\title{
Diagnostic Visualisation of Building Management System Energy Data
}

\author{
Paul Shabajee, Dan Schien and Chris Preist \\ Department of Computer Science \\ University of Bristol \\ Bristol, UK \\ paul.shabajee@bristol.ac.uk
}

\author{
John Brenton and Chris Jones \\ Sustainability, Estates Office \\ University of Bristol \\ Bristol, UK
}

\begin{abstract}
Index Terms - building management systems, energy management, visualization
\end{abstract}

\section{EXTENDED ABSTRACT}

This poster presents work done at the University of Bristol as part of the IODiCUS (Interoperable Open Digital Control Unit System) project [1]. The project is, in part, focused on exploring campus scale energy management and improving the usefulness of data from Building Management Systems (BMS) across the campus. Student halls of residence are in some respects comparable to social housing. A key aspect of that activity is to use BMS data to support and evaluate programmes that, for example, aim to help reduce overall energy use and reduce electricity demand during (expensive) peak periods.

Building Management Systems provide large volumes of fine grained and rich data about building operations and in particular energy use. For example, for electrical heating in student residential halls, a BMS provides device/room status every few minutes. Data available includes current temperature, thermostatic set-point, whether the device relay is 'on', operational 'program' (rules) that are active for the device and whether the program has been overridden by the resident. However, gaining actionable oversight and insight from such data can be problematic.

As part of the IODiCUS project [1] we have developed a prototype interactive diagnostic visualisation tool that uses BMS data to support the work of University of Bristol building facilities managers. The software behind the interface combines sensor data from the BMS with 'metadata' about the devices, (e.g. power rating) and buildings, (e.g. type of 'node' - room heater, kitchen heater, water heater, etc.). Using this integrated data, the interface provides the both oversight of energy use across a set of buildings ( $\mathrm{kWh})$ and interactive multidimensional filtering based on BMS data parameters and key temporal dimensions. Filtering enables the user to drill down to see energy use ( $\mathrm{kWh}$ ) of, for example, particular types of node, on particular days of the week, under specific operational programs, etc.

The interfaces are browser based and developed using open source software, including, d3.js (https://d3js.org/), Crossfilter (http://d3js.org/), dc.js (https://dc-js.github.io/dc.js/), bootstrap (http://getbootstrap.com/) \& jQuery (http://jquery.com/).

The tools enable facilities managers to carry out key diagnostic tasks - identified in consultation with University of Bristol Estates Department. Examples of diagnostic tasks supported include: i) obtaining an overview of energy use and energy using behaviours across device types, spaces/facilities and timescales, ii) identifying patterns, energy use 'hot spots' and variations in behaviours of buildings, spaces, equipment and residents, iii) evaluation of existing and piloted energy management initiatives and iv) spotting issues and anomalies that require further investigation.

The poster presents an illustrative example of the interface's ability to support facilities managers in investigating the effectiveness of BMS controlled energy reduction programs, in electrically heated student halls, aiming to reduce electricity use during the expensive, peak-demand period of weekday hours of $17-19 \mathrm{hrs}$.

The prototype system is continually being developed. Ongoing developments include: additions of building structure data as parameters, e.g. building name, storey, etc., and including other metrics including financial cost $(\mathfrak{E})$ and carbon footprint $(\mathrm{kgCO} 2 \mathrm{e})$

\section{REFERENCES}

[1] IODiCUS (2016) IODICUS Project. http://www.iodicus.org 